\title{
Patient and health provider costs of integrated HIV, diabetes and hypertension ambulatory health services in low-income settings - an empirical socio-economic cohort study in Tanzania and Uganda
}

\author{
Tinevimbo Shiri', Josephine Birungi ${ }^{2,3}$, Anupam V. Garrib', Sokoine L. Kivuyo ${ }^{4}$, Ivan Namakoola ${ }^{3}$,
} Janneth Mghamba ${ }^{5}$, Joshua Musinguzi ${ }^{5}$, Godfather Kimaro ${ }^{6}$, Gerald Mutungi ${ }^{7}$, Moffat J. Nyirenda ${ }^{2,8}$, Joseph Okebe ${ }^{1}$, Kaushik Ramaiya ${ }^{1,9}$, M. Bachmann ${ }^{10}$, Nelson K. Sewankambo ${ }^{11}$, Sayoki Mfinanga ${ }^{1,4,6}$, Shabbar Jaffar ${ }^{1 \dagger}$ and Louis W. Niessen ${ }^{1,12^{*}+}$ (D)

\begin{abstract}
Background: Integration of health services might be an efficient strategy for managing multiple chronic conditions in sub-Saharan Africa, considering the scope of treatments and synergies in service delivery. Proven to promote compliance, integration may lead to increased economies-of-scale. However, evidence on the socio-economic consequences of integration for providers and patients is lacking.

We assessed the clinical resource use, staff time, relative service efficiency and overall societal costs associated with integrating HIV, diabetes and hypertension services in single one-stop clinics where persons with one or more of these conditions were managed.

Methods: 2273 participants living with HIV infection, diabetes, or hypertension or combinations of these conditions were enrolled in 10 primary health facilities in Tanzania and Uganda and followed-up for up to 12 months. We collected data on resources used from all participants and on out-of-pocket costs in a sub-sample of 1531 participants, while a facility-level costing study was conducted at each facility. Health worker time per participant was assessed in a time-motion morbidity-stratified study among 228 participants. The mean health service cost per month and out-of-pocket costs per participant visit were calculated in 2020 US\$ prices. Nested bootstrapping from these samples accounted for uncertainties. A data envelopment approach was used to benchmark the efficiency of the integrated services. Last, we estimated the budgetary consequences of integration, based on prevalence-based projections until 2025, for both country populations.
\end{abstract}

\footnotetext{
* Correspondence: louis.niessen@lstmed.ac.uk

†Shabbar Jaffar and Louis W. Niessen contributed equally to this work.

'Liverpool School of Tropical Medicine, Pembroke Place, Liverpool L3 5QA,

UK

${ }^{12}$ Department of International Health, Johns Hopkins Bloomberg School of

Public Health, Baltimore, MD, USA

Full list of author information is available at the end of the article
}

(c) The Author(s). 2021 Open Access This article is licensed under a Creative Commons Attribution 4.0 International License, which permits use, sharing, adaptation, distribution and reproduction in any medium or format, as long as you give appropriate credit to the original author(s) and the source, provide a link to the Creative Commons licence, and indicate if changes were made. The images or other third party material in this article are included in the article's Creative Commons licence, unless indicated otherwise in a credit line to the material. If material is not included in the article's Creative Commons licence and your intended use is not permitted by statutory regulation or exceeds the permitted use, you will need to obtain permission directly from the copyright holder. To view a copy of this licence, visit http://creativecommons.org/licenses/by/4.0/ The Creative Commons Public Domain Dedication waiver (http://creativecommons.org/publicdomain/zero/1.0/) applies to the data made available in this article, unless otherwise stated in a credit line to the data. 
Results: Their average retention after 1 year service follow-up was 1911/2273 (84.1\%). Five hundred and eighty-two of 2273 (25.6\%) participants had two or all three chronic conditions and 1691/2273 (74.4\%) had a single condition. During the study, 84/2239 (3.8\%) participants acquired a second or third condition. The mean service costs per month of managing two conditions in a single participant were $\$ 39.11(95 \% \mathrm{Cl} 33.99,44.33), \$ 32.18(95 \% \mathrm{Cl} 30.35$, $34.07)$ and $\$ 22.65(95 \% \mathrm{Cl} 21.86,23.43)$ for the combinations of HIV and diabetes and of HIV and hypertension, diabetes and hypertension, respectively. These costs were $34.4 \%$ (95\% Cl 17.9\%, 41.9\%) lower as compared to managing any two conditions separately in two different participants. The cost of managing an individual with all three conditions was $48.8 \%$ (95\% Cl 42.1\%, 55.3\%) lower as compared to managing these conditions separately. Out-of-pocket healthcare expenditure per participant per visit was $\$ 7.33$ (95\% Cl 3.70, 15.86). This constituted 23.4\% $(95 \% \mathrm{Cl} 9.9,54.3)$ of the total monthly service expenditure per patient and $11.7 \%(95 \% \mathrm{Cl} 7.3,22.1)$ of their individual total household income. The integrated clinics' mean efficiency benchmark score was 0.86 (range 0.301.00) suggesting undercapacity that could serve more participants without compromising quality of care. The estimated budgetary consequences of managing multi-morbidity in these types of integrated clinics is likely to increase by $21.5 \%$ (range 19.2-23.4\%) in the next 5 years, including substantial savings of $21.6 \%$ on the provision of integrated care for vulnerable patients with multi-morbidities.

Conclusion: Integration of HIV services with diabetes and hypertension control reduces both health service and household costs, substantially. It is likely an efficient and equitable way to address the increasing burden of financially vulnerable households among Africa's ageing populations.

Additional economic evidence is needed from longer-term larger-scale implementation studies to compare extended integrated care packages directly simultaneously with evidence on sustained clinical outcomes.

Keywords: HIV control, Diabetes, Hypertension, Integrated care, Primary level, Economics, Efficiency, Vulnerable populations, Multi-morbidity

\section{Introduction}

The burden of non-communicable diseases (NCDs) has risen rapidly in Africa, and these diseases typically affect younger working populations than seen in developed countries [1-5]. Diabetes and hypertension alone are probably responsible for more than 2 million deaths a year on the continent [6]. Africa has a continuing high burden of HIV infection. Up until now, health services for HIV infection are organised in stand-alone clinics, separate from the rest of the health system, with separate channels for drug and diagnostic procurement and separate funding. The achievements of HIV programmes are impressive, with over $60 \%$ of people living with HIV infection in East and Southern Africa in regular care [7, 8]. In contrast, health care services for diabetes and hypertension, which are also often organised separately, are patchy, with only about $10-20 \%$ of people with these conditions estimated to be in care [9-11].

We evaluated the provision of an integrated health care service, via a 'one-stop' clinic, for a cohort of people living with HIV infection, diabetes, hypertension, or any combination of these conditions in Tanzania and Uganda at primary and secondary level to assess the acceptability of integrated care if compliance in HIV control was not affected negatively as was feared by country stake holders and health workers [12-15]. However, chronic conditions may lead households into catastrophic expenditure and deeply into poverty [16]. There are few data on the overall economic impact relating to provision of diabetes and hypertension services in Africa and to our knowledge none in relation to one-stop integrated care that specifically includes patients with multiple conditions [17-20].

We aimed to (1) measure the health service attendance and resource use, total time spend per patient and associated treatment costs in our cohort, as well as the additional out-of-pocket (OOP) costs per participant; (2) assess the relative efficiency of integrating services for HIV infection, diabetes and hypertension treatment; and (3) assess the imminent budgetary consequences of managing multi-morbidity in one-stop integrated clinics.

\section{Methods}

\section{Study design and participants}

The cohort study of integration of chronic care services, known as the Management of Chronic Conditions in Africa (MOCCA) study, has been described elsewhere [12]. In brief, 2416 individuals with either HIV, diabetes, hypertension, or combinations of these conditions were invited to join an integrated care clinic and 2273 were enrolled from 5th August 2018 and 21st May 2019, followed-up until the 30th of January 2020 [12-14]. The clinic delivered a single model of care, to patients regardless of condition. All patients were seen by the same clinical staff and shared a single reception, waiting areas and pharmacy, and systems for tracking and follow-up of patients, recording of clinical notes, counselling, 
appointments, patient cards and registrations. Where not available for patients with diabetes and hypertension, these were set up from new and aligned with those available for people living with HIV. At the onset, missing equipment and materials were supplemented. A refresher training for 1 day was carried out, also at study onset. Next, once-a-month continuous medication sessions were held on chronic conditions with health facility staff as part of their regular schedule. These aimed to ensure standardised clinical management in all three conditions. A small buffer supply of drugs was available to facilities in the event of there being interruptions to drug supplies.

We compared the total costs per patient with multimorbidities to the sum of treating the same conditions separately.

\section{Patient selection}

People were asked to become a participant in the cohort study on voluntary basis, as we assessed if integrated care were to be of 'no harm'. The number of patients with diabetes or with multiple conditions was limited; hence, all persons presenting with diabetes or with two or three of target conditions were invited consecutively to join. The numbers of people in care with HIV infection or with hypertension was large and so we sampled systematically, usually every 20th patient. In two small clinics, we enrolled patients known to study staff and invited them to the study. We excluded patients that were severely ill and needed referral to secondary care levels and patients that would not continue to live in the area.

\section{Socio-economic study}

The embedded economic sub-study adopted both health service and patient perspectives, that is, a broad societal perspective. A costing study of delivering services for HIV, diabetes and hypertension was conducted in each participating facility. Individual resource use was recorded in all participants in the overall clinical questionnaire. We collected detailed patient cost information on the volume of resources in 1531 patients and their households, shown in Fig. 1, on the second row. This figure shows that in Tanzania 1041 patients were included and in Uganda 1375. The lowest number of participants (below 50) was in the group of participants with both HIV and diabetes and the group with the three target conditions. All other groups had more than

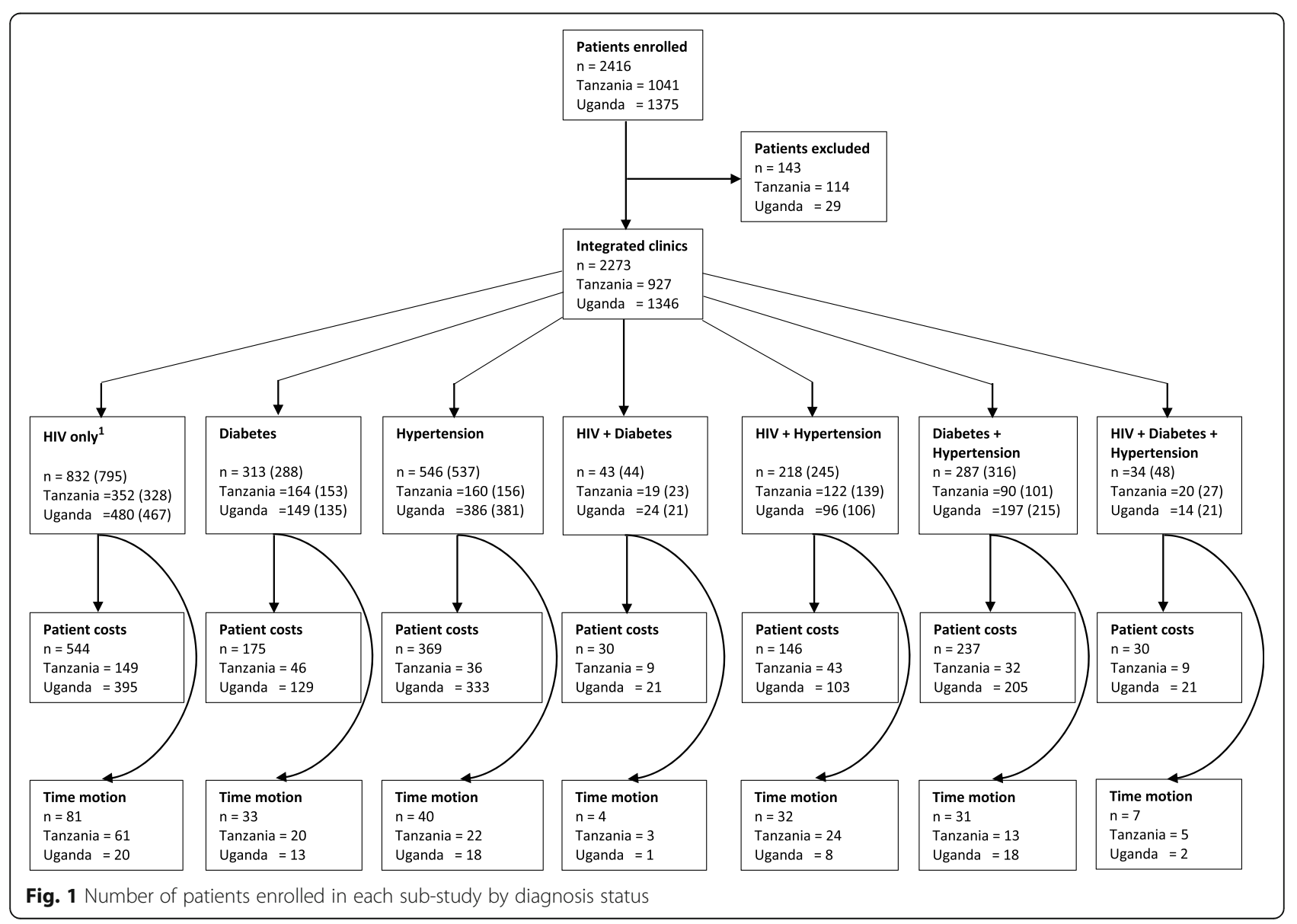


200 participants with the HIV as the largest with 832 participants.

We recorded the diagnostic tests performed and the drugs prescribed, individually. Unit costs for both individual drugs and diagnostic tests were available at the facility level, based on suppliers' invoices, and where they were not available, local market prices were adopted. Unit costs and specific diagnostic tests are shown in Tables A1 and A2, respectively, in the Additional file 1. The direct and indirect costs were prospectively measured in the sub-sample of 1531 participants, recruited consecutively. Also, a sub-sample of 228 participants was enrolled randomly in a comorbidity-stratified timemotion study to assess health worker time spent per participant per visit (Fig. 1).

\section{Collection of data on integrated programme overheads}

A standardised costing tool [21] was administered to estimate facility level costs in each of the 10 facilities, both in Uganda and Tanzania. It collected information on numbers and level of facility health staff to estimate salary and related costs of all staff involved in integrated service delivery. This was supplemented with information on numbers of other staff involved in caring for the participant, office supplies, travel expenses, utility bills, medication, medical consumables and supplies, number of laboratory testing, medical equipment, furniture, vehicles and rental space used for client care. Rental costs were based on the total area of the space utilised and the average cost per square metre in each setting. Routine information on total patient numbers at each facility every month was collected to facilitate the attribution of the integrated care service. The supply of drugs and diagnostic tests was variable. Some of the facilities had insufficient drug supply, inadequate functional laboratory equipment to conduct tests and some lacked the equipment and or reagents to conduct some laboratory tests (Table A3 in the Additional file 1). We therefore calculated the health service cost per patient at each facility based on the observed use of resources and assumed a full month's supply of drugs and diagnostics per each supply round.

\section{Collection of data on participant-related out-of-pocket costs}

We administered a patient cost questionnaire to a subsample of participants, sequentially (Fig. 1). Information collected included participant income, total household income, demographic characteristics, medical and nonmedical costs prior to attending to the health care facility and on the day of attendance, loss of earnings due to participants attending a health care facility and details on health care financing such as if the patients had to borrow money or sell valuables to be able to attend the healthcare facility. The participant's reported monthly income was used to calculate the value of hours lost. In addition, we collected information on the participants' travel time to the health facility, transport costs, costs of meals and other out-of-pocket payments such as payments for health services and medications.

\section{Time-motion study involving health workers and participants}

In our time-motion study, we recorded the time each type of health facility staff spent providing care and the time that participants spent waiting for each component of health care. The total time spent at the facility by participants was added to their travelling time to estimate productivity losses related to their visit (see annex for further details).

\section{Statistical analysis}

In an ingredient approach to collect resource use data [22], following consolidated health economic evaluation reporting standards (CHEERS) guidelines [23], we calculated total health service cost and out-of-pocket cost per participant by comorbidity category. We assigned costs related to drugs, diagnostics, programme costs (such as staff salaries, administration, furniture and equipment), transport and other out-of-pocket expenses, including total loss of productivity due to time spent visiting the facility. We used non-parametric bootstrapping [24] to assess the uncertainties which were reported as $95 \%$ confidence intervals $(95 \% \mathrm{CI})$. All costs were calculated in 2020 US\$ prices. The estimated participant and programme overhead costs for facility-level management of diabetes and hypertension treatment were suboptimal due to shortages of medications and staff. We calculated the actual real-life cost and re-assigned the overheads and personnel costs calculated for the people living with HIV infection to those living with diabetes and hypertension. The number of drug and diagnostic tests was based on the actual reported resources used by the participants.

We present here aggregate and country-based uncertainty distributions of clinical resource use within each subgroup group and across groups. These are determined by the health guidelines used in the training and reinforced by repeat and continuous education sessions. These estimates document the complete range of services cost per individual and allows for a comparison of distributions between the various types of patients, in the diverse settings.

To assess and to benchmark the efficiency of the integrated clinics, we applied an input-output data envelopment analysis (DEA) [25-27] to measure the relative efficiency of HIV clinics, NCD clinics and outpatient 
departments that manage HIV, diabetes and hypertension in Uganda and Tanzania.

The efficiency of a facility is defined as the ratio of the weighted sum of outputs (total virtual output) to the weighted sum of inputs (total virtual input), with the weights being obtained in favour of each evaluated facility to measure the optimisation of activities processes. Each facility is using multiple inputs to produce multiple outputs. We denote a vector of inputs for so-called decision-making unit (DMUs) as well as a vector of outputs is outputs. The model [27] is formulated and solved for each hospital to obtain its efficiency score, once relative weights associated with the inputs and the outputs are identified and valued, respectively. These weights are calculated in a manner that they provide the highest possible efficiency score for each facility under evaluation [27].

We standardised key outputs given key resource inputs of the facilities. In the computation, we re-scaled the included output metrics between 0 and 1 . A score of 1 equals the maximum outputs score observed among the individual clinics, given the input levels. We included the number of participants attended and diagnostic tests, and medicines provided were used as the output benchmarks. The number of key clinical staff was used as input variables, categorised into three groups: clinicians, nurses and other health workers which included laboratory technicians and pharmacists. We excluded the use of health services or patient costs data, as these differ between the two counties. The outputs were specified as (1) the staff workload defined as the number of full-time staff divided by the number of daily participant visits, (2) laboratory tests per participant defined as the number of daily laboratory tests divided by the number of daily participant visits and (3) drug availability defined by the proportion of patients who receive drugs at no costs from the facilities before stock-outs occur.

We used the empirical integration cost estimates in a future forecasting for the two individual country settings, based on DEA results and prevalence-based modelling accounting for shorter survival and including the observed uncertainty ranges of the total cost per patient profile. We conservatively assumed that prevalence of these - multiple - conditions will remain constant and independent over time (see Additional file 1 for per country details). The source of the country population projections is United Nations Population Council [13]. All analyses were conducted using SAS version 9.4 (SAS Institute, Inc., NC). The necessary assumption here is that the estimates from the study need to be representative for similar situations, elsewhere in the two countries. Presently, this cannot be verified, and the estimates need to be viewed at as 'what-if' scenarios. The uncertainties in the presented changes are more driven by the uncertainties in the demographic-epidemiological data and included the narrow uncertainty ranges of the collected cost per patient categories. Conservatively, we present the relative changes in the health service cost projections.

\section{Results \\ Study participation}

Their retention in care after 1 year follow-up was 1911/ 2273 (84.1\%). At enrolment, 582/2273 (25.6\%) participants had 2 or all 3 chronic conditions of study (referred to hereon as multi-morbidity) while the remainder 1691/ $2273(74.4 \%)$ had a single condition. Over the course of the study, an additional 84/2239 (3.8\%) participants acquired a second or third condition. Figure 1 shows the flow diagram of participants in the economic study.

Baseline characteristics for individuals sampled to provide participant out-of-pocket expenses are shown in Table 1. Two-thirds or more were women in each disease category. Overall, half (529/1050) were selfemployed and 19\% (284/1504) reported no income. Most participants $(78 \%$; 1159/1481) used public transport to access health facilities. Strikingly, over a third of the participants $(563 / 1492)$ left a young child below the age of 6 years at home and 10\% (149/1510) asked a family member or friend to accompany them to the health care facility.

Table 2 shows the resources used by participants. Overall, 1464 of 1620 (90.4\%) participants with a single condition and 619 of $653(94.8 \%)$ with 2 or all 3 conditions were on treatment for their health conditions. Of the diagnostic tests, the most common resources were fasting blood glucose and the plasma viral load.

\section{Service use and costs of integrated care services for HIV infection, diabetes and hypertension}

The mean health service cost per participant per month based on their individual resources used, assuming a full month's patient supply of drugs and diagnostics, and assigning the overheads and personnel costs calculated for the people living with HIV infection to those living with diabetes and hypertension are shown in Table 3. In this approach, the costs of medications and of diagnostic tests per patient per month were higher for HIV infection than the additions of costs for diabetes or hypertension in individuals with single conditions (HIV infection alone, diabetes alone and hypertension alone). For participants who had multi-morbidity, their monthly drug and diagnostic costs were simply the addition of drug and diagnostic costs of individual conditions. Facilitybased personnel costs were similar across all conditions.

The added monthly costs of managing either hypertension or diabetes among participants living with HIV infection were $-\$ 2.03$ (95\% CI $-4.33,0.27)$ and $+\$ 4.89$ 
Table 1 The characteristics of individuals who were enrolled for patient-related costs by disease condition. Values are presented as $\%$ (n), with the denominator given by the total count with a response for that characteristic, except for average group age.

\begin{tabular}{|c|c|c|c|c|c|c|c|c|c|}
\hline Characteristics & Category & Overall & HIV & Diabetes & Hypertension & $\begin{array}{l}\text { HIV + } \\
\text { diabetes }\end{array}$ & $\begin{array}{l}\text { HIV + } \\
\text { hypertension }\end{array}$ & $\begin{array}{l}\text { Diabetes + } \\
\text { hypertension }\end{array}$ & $\begin{array}{l}\text { HIV + diabetes } \\
+ \text { hypertension }\end{array}$ \\
\hline Age & Average (range) & & $38(18-78)$ & $49(19-77)$ & $56(27-86)$ & & & $5(21-85)$ & \\
\hline \multirow[t]{3}{*}{ Gender } & Total & 1531 & 544 & 175 & 369 & 30 & 146 & 237 & 30 \\
\hline & Female & 71.5 (1094) & $68.0(370)$ & $67.4(118)$ & $75.3(278)$ & $66.7(20)$ & $70.5(103)$ & $76.4(181)$ & $80.0(24)$ \\
\hline & Male & $28.5(437)$ & $32.0(174)$ & $32.6(57)$ & $24.7(91)$ & $33.3(10)$ & $29.5(43)$ & $23.6(56)$ & $20.0(6)$ \\
\hline \multirow[t]{5}{*}{ Highest education level } & Total & 1051 & 407 & 138 & 235 & 18 & 90 & 142 & 21 \\
\hline & No formal education & $9.4(99)$ & $5.9(24)$ & $12.3(17)$ & $9.8(23)$ & $11.1(2)$ & $7.8(7)$ & $15.5(22)$ & $19.0(4)$ \\
\hline & Primary education & $61.7(648)$ & $62.4(254)$ & $61.6(85)$ & $62.1(146)$ & $50.0(9)$ & $62.2(56)$ & $61.3(87)$ & $52.4(11)$ \\
\hline & Secondary education & $22.4(235)$ & $24.6(100)$ & $18.1(25)$ & $21.7(51)$ & $27.8(5)$ & $26.7(24)$ & $16.9(24)$ & $28.6(6)$ \\
\hline & Higher education & $6.6(69)$ & $7.1(29)$ & $8.0(11)$ & $6.4(15)$ & $11.1(2)$ & $3.3(3)$ & $6.3(9)$ & $0.0(0)$ \\
\hline \multirow[t]{6}{*}{ Marital status } & Total & 1053 & 407 & 138 & 236 & 18 & 90 & 143 & 21 \\
\hline & Currently married & $51.0(537)$ & $39.8(162)$ & $68.8(95)$ & $57.2(135)$ & $61.1(11)$ & $47.8(43)$ & $58.0(83)$ & $38.1(8)$ \\
\hline & Separated & $15.6(164)$ & $17.9(73)$ & $13.0(18)$ & $17.4(41)$ & $5.6(1)$ & $11.1(10)$ & $14.0(20)$ & $4.8(1)$ \\
\hline & Widowed & $14.9(157)$ & $9.8(40)$ & $8.0(11)$ & $18.6(44)$ & $11.1(2)$ & $26.7(24)$ & $17.5(25)$ & $52.4(11)$ \\
\hline & Never married & $13.6(143)$ & $25.1(102)$ & $9.4(13)$ & $4.7(11)$ & 16.7 (3) & $4.4(4)$ & $7.0(10)$ & $0.0(0)$ \\
\hline & Divorced & $4.9(52)$ & $7.4(30)$ & $0.7(1)$ & $2.1(5)$ & $5.6(1)$ & $10.0(9)$ & $3.5(5)$ & $4.8(1)$ \\
\hline \multirow{10}{*}{$\begin{array}{l}\text { Participant's main } \\
\text { occupation }\end{array}$} & Total & 1050 & 405 & 138 & 236 & 18 & 90 & 142 & 21 \\
\hline & Self-employed & $50.4(529)$ & $51.1(207)$ & $51.4(71)$ & $48.3(114)$ & $50.0(9)$ & $51.1(46)$ & $50.0(71)$ & $52.4(11)$ \\
\hline & Casual labourer & $11.7(123)$ & $17.3(70)$ & $5.8(8)$ & $8.9(21)$ & 16.7 (3) & $6.7(6)$ & $10.6(15)$ & $0.0(0)$ \\
\hline & $\begin{array}{l}\text { Non-governmental } \\
\text { organisation } \\
\text { employee }\end{array}$ & $10.2(107)$ & $11.1(45)$ & $10.1(14)$ & $12.7(30)$ & $16.7(3)$ & $8.9(8)$ & $4.9(7)$ & $0.0(0)$ \\
\hline & $\begin{array}{l}\text { Unemployed (able to } \\
\text { work) }\end{array}$ & $9.5(100)$ & $8.6(35)$ & 10.9 (15) & $7.2(17)$ & $5.6(1)$ & $7.8(7)$ & $16.2(23)$ & $9.5(2)$ \\
\hline & Housewife & $7.5(79)$ & $5.9(24)$ & $10.9(15)$ & $6.4(15)$ & $5.6(1)$ & $12.2(11)$ & $7.0(10)$ & $14.3(3)$ \\
\hline & $\begin{array}{l}\text { Unemployed (unable } \\
\text { to work) }\end{array}$ & $5.0(52)$ & $2.2(9)$ & $2.2(3)$ & $9.3(22)$ & $0.0(0)$ & $7.8(7)$ & $5.6(8)$ & $14.3(3)$ \\
\hline & $\begin{array}{l}\text { Government } \\
\text { employee }\end{array}$ & $2.9(30)$ & $1.2(5)$ & $4.3(6)$ & $4.7(11)$ & $5.6(1)$ & $3.3(3)$ & $2.8(4)$ & $0.0(0)$ \\
\hline & Retired & $2.3(24)$ & $1.5(6)$ & $3.6(5)$ & $2.5(6)$ & $0.0(0)$ & $2.2(2)$ & $2.1(3)$ & $9.5(2)$ \\
\hline & Student & $0.6(6)$ & $1.0(4)$ & $0.7(1)$ & $0.0(0)$ & $0.0(0)$ & $0.0(0)$ & $0.7(1)$ & $0.0(0)$ \\
\hline \multirow{7}{*}{$\begin{array}{l}\text { Individual who } \\
\text { contributes most income } \\
\text { in the household }\end{array}$} & Total & 1525 & 544 & 175 & 363 & 30 & 146 & 237 & 30 \\
\hline & Self & $57.8(881)$ & $62.5(340)$ & $52.0(91)$ & $54(196)$ & $66.7(20)$ & $61.0(89)$ & $53.6(127)$ & $60.0(18)$ \\
\hline & Spouse & $19.4(296)$ & $19.3(105)$ & $26.9(47)$ & $18.5(67)$ & $20.0(6)$ & $13.0(19)$ & $20.7(49)$ & $10.0(3)$ \\
\hline & Children & $13.9(212)$ & $3.5(19)$ & $10.9(19)$ & $22.0(80)$ & $3.3(1)$ & $19.9(29)$ & $23.2(55)$ & $30.0(9)$ \\
\hline & Brother/sister & $4.1(63)$ & $6.8(37)$ & $4.6(8)$ & $2.8(10)$ & $6.7(2)$ & $2.1(3)$ & $1.3(3)$ & $0.0(0)$ \\
\hline & Other & $3.0(46)$ & $4.4(24)$ & $2.9(5)$ & $2.8(10)$ & $0.0(0)$ & $2.7(4)$ & $1.3(3)$ & $0.0(0)$ \\
\hline & Parent & $1.8(27)$ & $3.5(19)$ & $2.9(5)$ & $0.0(0)$ & $3.3(1)$ & $1.4(2)$ & $0.0(0)$ & $0.0(0)$ \\
\hline \multirow{7}{*}{$\begin{array}{l}\text { Main occupation of the } \\
\text { person who contributes } \\
\text { most in the household }\end{array}$} & Total & 770 & 265 & 100 & 177 & 16 & 74 & 123 & 15 \\
\hline & Self-employed & 55.7 (429) & $52.5(139)$ & $56.0(56)$ & $59.3(105)$ & $50.0(8)$ & $51.4(38)$ & $60.2(74)$ & $60.0(9)$ \\
\hline & Professional & $24.2(186)$ & $23.4(62)$ & $26.0(26)$ & $25.4(45)$ & $31.3(5)$ & $23.0(17)$ & $22.0(27)$ & 26.7 (4) \\
\hline & Other & $10.0(77)$ & $13.2(35)$ & $7.0(7)$ & $7.9(14)$ & $0.0(0)$ & $14.9(11)$ & $6.5(8)$ & $13.3(2)$ \\
\hline & Farmer & $6.1(47)$ & $6.4(17)$ & $7.0(7)$ & $5.1(9)$ & $18.8(3)$ & $2.7(2)$ & $7.3(9)$ & $0.0(0)$ \\
\hline & Unemployed & $3.0(23)$ & $4.2(11)$ & $1.0(1)$ & $1.1(2)$ & $0.0(0)$ & $5.4(4)$ & $4.1(5)$ & $0.0(0)$ \\
\hline & Housewife & $1.0(8)$ & $0.4(1)$ & $3.0(3)$ & $1.1(2)$ & $0.0(0)$ & $2.7(2)$ & $0.0(0)$ & $0.0(0)$ \\
\hline \multirow{2}{*}{$\begin{array}{l}\text { Participant's main income } \\
\text { is based on }\end{array}$} & Total & 1504 & 538 & 171 & 361 & 30 & 140 & 235 & 29 \\
\hline & Business earnings & 23.7 (356) & $22.1(119)$ & $26.9(46)$ & $22.7(82)$ & $33.3(10)$ & $22.9(32)$ & $24.3(57)$ & $34.5(10)$ \\
\hline
\end{tabular}


Table 1 The characteristics of individuals who were enrolled for patient-related costs by disease condition. Values are presented as $\%(n)$, with the denominator given by the total count with a response for that characteristic, except for average group age. (Continued)

\begin{tabular}{|c|c|c|c|c|c|c|c|c|c|}
\hline Characteristics & Category & Overall & HIV & Diabetes & Hypertension & $\begin{array}{l}\text { HIV + } \\
\text { diabetes }\end{array}$ & $\begin{array}{l}\text { HIV + } \\
\text { hypertension }\end{array}$ & $\begin{array}{l}\text { Diabetes + } \\
\text { hypertension }\end{array}$ & $\begin{array}{l}\text { HIV + diabetes } \\
+ \text { hypertension }\end{array}$ \\
\hline & Daily wage & $27.4(412)$ & $32.7(176)$ & $26.3(45)$ & $19.9(72)$ & $16.7(5)$ & $25.7(36)$ & $31.1(73)$ & $17.2(5)$ \\
\hline & Have no income & $18.9(284)$ & $16.0(86)$ & $15.8(27)$ & $24.4(88)$ & $16.7(5)$ & $21.4(30)$ & $17.4(41)$ & $24.1(7)$ \\
\hline & Monthly salary & $16.9(254)$ & $19.3(104)$ & $15.8(27)$ & $18.3(66)$ & $16.7(5)$ & $15.7(22)$ & $11.5(27)$ & $10.3(3)$ \\
\hline & Sale of farm produce & $7.8(117)$ & $5.6(30)$ & $7.0(12)$ & $8.9(32)$ & $10.0(3)$ & $10.0(14)$ & $10.6(25)$ & $3.4(1)$ \\
\hline & Other & $5.4(81)$ & $4.3(23)$ & $8.2(14)$ & $5.8(21)$ & $6.7(2)$ & $4.3(6)$ & $5.1(12)$ & $10.3(3)$ \\
\hline \multirow{5}{*}{$\begin{array}{l}\text { Mode of transport used } \\
\text { today }\end{array}$} & Total & 1481 & 526 & 169 & 358 & 29 & 137 & 234 & 28 \\
\hline & Public transport & $78.3(1159)$ & 83.5 (439) & $75.7(128)$ & $66.8(239)$ & $93.1(27)$ & $88.3(121)$ & $76.1(178)$ & $96.4(27)$ \\
\hline & Walking & $19.5(289)$ & $14.8(78)$ & $21.3(36)$ & $30.2(108)$ & $6.9(2)$ & $10.9(15)$ & $21.4(50)$ & $0.0(0)$ \\
\hline & Personal car & $1.9(28)$ & $1.3(7)$ & $3.0(5)$ & $2.2(8)$ & $0.0(0)$ & $0.7(1)$ & $2.6(6)$ & $3.6(1)$ \\
\hline & Other & $0.3(5)$ & $0.4(2)$ & $0.0(0)$ & $0.8(3)$ & $0.0(0)$ & $0.0(0)$ & $0.0(0)$ & $0.0(0)$ \\
\hline \multirow{3}{*}{$\begin{array}{l}\text { Number that left young } \\
\text { children (aged less than } \\
\text { about } 6 \text { years) at home }\end{array}$} & Total & 1492 & 533 & 169 & 360 & 30 & 138 & 233 & 29 \\
\hline & Yes & $37.7(563)$ & $34.5(184)$ & $40.8(69)$ & $38.9(140)$ & $33.3(10)$ & $34.1(47)$ & $43.8(102)$ & $37.9(11)$ \\
\hline & No & $62.3(929)$ & $65.5(349)$ & $59.2(100)$ & $61.1(220)$ & $66.7(20)$ & $65.9(91)$ & $56.2(131)$ & $62.1(18)$ \\
\hline \multirow{3}{*}{$\begin{array}{l}\text { Number who had to ask } \\
\text { someone to accompany } \\
\text { them }\end{array}$} & Total & 1510 & 537 & 174 & 363 & 30 & 143 & 233 & 30 \\
\hline & Yes & $9.9(149)$ & $7.8(42)$ & $12.6(22)$ & $11.8(43)$ & $6.7(2)$ & $7.7(11)$ & $11.6(27)$ & $6.7(2)$ \\
\hline & No & $90.1(1361)$ & $92.2(495)$ & $87.4(152)$ & $88.2(320)$ & $93.3(28)$ & $92.3(132)$ & $88.4(206)$ & $93.3(28)$ \\
\hline
\end{tabular}

(95\% CI - 0.36, 10.22) per participant, respectively, compared to managing HIV only (Table 3 ). For people living with diabetes, the additional cost of managing hypertension was $\$ 4.37$ (95\% CI 3.27, 5.49) per participant per month, compared to managing diabetes only.

The mean health service costs of managing two conditions in one participant were $\$ 39.11$ (95\% CI 33.99, 44.33), $\$ 32.18$ (95\% CI 30.35, 34.07) and $\$ 22.65$ (95\% CI 21.86, 23.43) for HIV and diabetes, HIV and hypertension, diabetes and hypertension per month, respectively. These costs were $34.4 \%$ (95\% CI 17.9\%, 41.9\%) lower than managing two conditions separately in two different participants. The cost of managing an individual with all three conditions was $\$ 36.38$ (95\% CI 31.83, 41.07) per month, which was $48.8 \%$ (95\% CI $42.1 \%$, $55.3 \%)$ lower than managing 3 conditions separately in three different participants.

\section{Participant-related household expenditure}

The overall mean participant-related cost was $\$ 7.33$ (95\% CI 3.70, 15.86) per visit, and this comprised consultation costs, transport costs, medication costs, lost labour and other out-of-pocket costs (Table 4). The mean transport costs were $\$ 1.44$ (95\% CI 0.81, 2.18) for a return trip to health facilities per visit in the two countries, which amounts to $4.5 \%$ (95\% CI 2.1, 9.6) of the participant's total household income. Mean medication costs of $\$ 2.25$ (95\% CI $0.00,7.55)$ per participant per month were the main drivers of patient-related costs, mainly for participants without HIV. Overall, participant-related costs per visit, constituted $11.7 \%$
(95\% CI 7.3, 22.1) of the monthly total household earnings.

Participants also reported losing earnings from visiting the facilities. This loss was nearly as much as their twoway transport costs (Supplementary Table A4 in the Additional file 1). The mean travelling time to reach the facilities was $45 \mathrm{~min}$ in Tanzania and $30 \mathrm{~min}$ in Uganda, which translates to a mean of $\$ 0.78$ (95\% CI $0.41,1.31$ ) per return trip economic cost for loss of earnings or productivity.

All in all, out-of-pocket healthcare expenditure per health care visit comprised $23.4 \%$ (95\% CI 9.9, 54.3) of the total cost of integrated care (i.e., the sum of health service and patient-related costs).

\section{Staff- and patient-related time and time costs per visit}

In our estimates, we used the observed time spent by health care staff members to provide patient care and calculated the involved costs per visit (Table 5). Clinicians spent the most time devoted to participant care, with a mean $15.3(95 \%$ CI $11.4,20.3)$ min per participant. The staff time, hence, staff cost for providing care to individuals with multiple conditions was similar or was slightly higher but not significantly different from the staff cost of caring for individuals with a single condition. Clinicians took a mean time of 15.3 (95\% CI 11.9, 19.6) min per participant with a single condition and 15.4 (95\% CI 11.1, 20.9) min for a participant with multiple conditions, nurses a mean time of 11.1 (95\% CI 6.3, 16.2) and 13.8 (95\% CI 5.4, 33.7) $\min$ and laboratory 
Table 2 Resource use description showing the proportion of individuals who were on medication and the number of laboratory tests performed during the study follow-up

\begin{tabular}{|c|c|c|c|c|c|c|c|}
\hline Resource & HIV & Diabetes & Hypertension & $\begin{array}{l}\text { HIV + } \\
\text { diabetes }\end{array}$ & $\begin{array}{l}\text { HIV + } \\
\text { hypertension }\end{array}$ & $\begin{array}{l}\text { Diabetes + } \\
\text { hypertension }\end{array}$ & $\begin{array}{l}\text { HIV + diabetes + } \\
\text { hypertension }\end{array}$ \\
\hline $\begin{array}{l}\text { Number at study } \\
\text { end }\end{array}$ & 795 & 288 & 537 & 44 & 245 & 316 & 48 \\
\hline \multicolumn{8}{|l|}{ Medication, $n(\%)$} \\
\hline No medication & $\begin{array}{l}76 \\
(9.6 \%)\end{array}$ & $31(10.8 \%)$ & $49(9.1 \%)$ & $1(2.3 \%)$ & $18(7.3 \%)$ & $14(4.4 \%)$ & $1(2.1 \%)$ \\
\hline \multirow[t]{7}{*}{ On medication } & $\begin{array}{l}719 \\
(90.4 \%)\end{array}$ & $\begin{array}{l}\text { Overall, } 257 \\
(89.2 \%)\end{array}$ & $\begin{array}{l}\text { Overall, } 488 \\
(90.9 \%)\end{array}$ & $\begin{array}{l}\text { Both drugs, } 29 \\
(65.9 \%)\end{array}$ & $\begin{array}{l}\text { Both drugs, } 131 \\
(53.5 \%)\end{array}$ & $\begin{array}{l}\text { Both drugs, } 239 \\
(75.6 \%)\end{array}$ & All drugs, 26 (54.2\%) \\
\hline & & $\begin{array}{l}1 \text { drug, } 104 \\
(36.1 \%)\end{array}$ & $\begin{array}{l}1 \text { drug, } 115 \\
(21.4 \%)\end{array}$ & $\begin{array}{l}\text { ART only, } 10 \\
(22.7 \%)\end{array}$ & $\begin{array}{l}\text { ART only, } 87 \\
(35.5 \%)\end{array}$ & $\begin{array}{l}\text { Diabetic only, } 40 \\
(12.7 \%)\end{array}$ & ART only, 8 (16.7\%) \\
\hline & & $\begin{array}{l}2 \text { drugs, } 144 \\
(50.0 \%)\end{array}$ & $\begin{array}{l}2 \text { drugs, } 282 \\
(52.5 \%)\end{array}$ & $\begin{array}{l}\text { Diabetic only, } 4 \\
(9.1 \%)\end{array}$ & $\begin{array}{l}\text { Hypertensive only, } \\
9(3.7 \%)\end{array}$ & $\begin{array}{l}\text { Hypertensive only, } \\
23(7.3 \%)\end{array}$ & Diabetic only, 1 (2.1\%) \\
\hline & & $\begin{array}{l}3 \text { drugs, } 9 \\
(3.1 \%)\end{array}$ & $\begin{array}{l}3 \text { drugs, } 91 \\
(17.0 \%)\end{array}$ & & & & $\begin{array}{l}\text { Hypertensive only, } 2 \\
(4.2 \%)\end{array}$ \\
\hline & & & & & & & $\begin{array}{l}\text { ART and diabetic, } 6 \\
(12.5 \%)\end{array}$ \\
\hline & & & & & & & $\begin{array}{l}\text { ART and hypertensive, } 3 \\
(6.3 \%)\end{array}$ \\
\hline & & & & & & & $\begin{array}{l}\text { Hypertensive and } \\
\text { diabetic, } 1(2.1 \%)\end{array}$ \\
\hline \multicolumn{8}{|l|}{ Diagnostics, $n(\%)^{a}$} \\
\hline Viral load test & $\begin{array}{l}597 \\
(75.1)\end{array}$ & $0(0.0)$ & $0(0.0)$ & $34(77.3)$ & $160(65.3)$ & $0(0.0)$ & $31(64.6)$ \\
\hline CD4 count test & $\begin{array}{l}87 \\
(10.9)\end{array}$ & $0(0.0)$ & $0(0.0)$ & $2(4.5)$ & $23(9.4)$ & $0(0.0)$ & $3(6.3)$ \\
\hline $\mathrm{HbA1c}$ test & $7(0.9)$ & $171(59.4)$ & $4(0.7)$ & $22(50.0)$ & $2(0.8)$ & $130(41.1)$ & $22(45.8)$ \\
\hline $\begin{array}{l}\text { Fasting blood } \\
\text { sugar test }\end{array}$ & $33(4.2)$ & $273(94.8)$ & $45(8.4)$ & $34(77.3)$ & $10(4.1)$ & $300(94.9)$ & $42(87.5)$ \\
\hline $\begin{array}{l}\text { Random blood } \\
\text { sugar test }\end{array}$ & $48(6.0)$ & $104(36.1)$ & $42(7.8)$ & $13(29.5)$ & $12(4.9)$ & $130(41.1)$ & $16(33.3)$ \\
\hline Haemoglobin test & $41(5.2)$ & $7(2.4)$ & $5(0.9)$ & $2(4.5)$ & $12(4.9)$ & $2(0.6)$ & $3(6.3)$ \\
\hline Cholesterol test & $6(0.8)$ & $34(11.8)$ & $28(5.2)$ & $2(4.5)$ & $6(2.4)$ & $26(8.2)$ & $1(2.1)$ \\
\hline Creatinine test & $14(1.8)$ & $25(8.7)$ & $19(3.5)$ & $3(6.8)$ & $4(1.6)$ & $22(7.0)$ & $1(2.1)$ \\
\hline Urine test & $7(0.9)$ & $11(3.8)$ & $15(2.8)$ & $2(4.5)$ & $5(2.0)$ & $17(5.4)$ & $2(4.2)$ \\
\hline $\begin{array}{l}\text { Renal and liver } \\
\text { function test }\end{array}$ & $4(0.5)$ & $16(5.6)$ & $20(3.7)$ & $3(6.7)$ & $13(6.1)$ & $41(13.0)$ & $3(6.3)$ \\
\hline Other tests ${ }^{\mathrm{b}}$ & $73(9.2)$ & $63(21.9)$ & $62(11.5)$ & $14(31.1)$ & $43(20.2)$ & $62(19.7)$ & $6(12.5)$ \\
\hline
\end{tabular}

${ }^{a}$ Number of patients with at least a test performed during the follow-up period

${ }^{b}$ Other tests include tests such as pregnancy test, syphilis, malaria test, etc.

technicians 9.2 (95\% CI 4.3, 17.3) and 11.5 (95\% CI 7.0, 22.0) min, respectively.

The mean total time participants took at the facility (i.e., from when they registered their arrival at the health facility, until they left the facility care) was 145.8 (95\% CI 86.0, 184.7) min per patient. We observed that this time estimate was not significantly different for a visit among participants with a single condition (mean time of $150.3(95 \%$ CI $126.3,176.6) \mathrm{min}$ ) and multiple conditions (mean time of 142.4 (95\% CI 74.6, 189.8) $\mathrm{min}$ ).
This observed time translates to a mean loss of participant productivity of $\$ 1.42$ (95\% CI $0.85,2.42)$ per participant per visit based on the estimated participant's earnings per month (Table 4)

Overall, the lowest percentage reduction in monthly income occurred among people with HIV only and those with only hypertension, i.e., $9.3 \%(8.3,10.5)$ and, respectively $9.2 \%(7.5,11.1)$. This was higher among people with combinations of conditions, HIV and diabetes, HIV and hypertension, and hypertension and diabetes. This 
Table 3 The monthly health service cost per patient of managing individuals with one, two and three conditions in an integrated one-stop clinic in Tanzania and Uganda. The mean costs and 95\% confidence intervals reported are in 2020 US\$

\begin{tabular}{|c|c|c|c|c|c|c|c|}
\hline Cost component & HIV only & Diabetes & Hypertension & $\begin{array}{l}\text { HIV + } \\
\text { diabetes }{ }^{1 \#}\end{array}$ & $\begin{array}{l}\text { HIV + } \\
\text { hypertension }\end{array}$ & $\begin{array}{l}\text { Diabetes + } \\
\text { hypertension }\end{array}$ & $\begin{array}{l}\text { HIV + diabetes + } \\
\text { hypertension }\end{array}$ \\
\hline $\begin{array}{l}\text { Total monthly } \\
\text { cost }\end{array}$ & $\begin{array}{l}\$ 34.21(32.92, \\
35.59)\end{array}$ & $\begin{array}{l}\$ 18.28(17.52 \\
19.04)\end{array}$ & $\begin{array}{l}\$ 18.60(18.13 \\
19.08)\end{array}$ & $\begin{array}{l}\$ 39.11(33.99 \\
44.33)\end{array}$ & $\begin{array}{l}\$ 32.18(30.35 \\
34.07)\end{array}$ & $\$ 22.65(21.86,23.43)$ & $\$ 36.38(31.83,41.07)$ \\
\hline Personnel & $\begin{array}{l}\$ 7.36(7.21 \\
7.52)\end{array}$ & $\begin{array}{l}\$ 7.34(7.08 \\
7.61)\end{array}$ & $\begin{array}{l}\$ 8.15(7.99 \\
8.32)\end{array}$ & $\begin{array}{l}\$ 6.71(6.15 \\
7.26)\end{array}$ & $\begin{array}{l}\$ 6.62(6.36, \\
6.90)\end{array}$ & $\$ 7.72(7.49,7.94)$ & $\$ 6.37(5.80,6.95)$ \\
\hline Medication & $\begin{array}{l}\$ 12.21(11.60 \\
12.83)\end{array}$ & $\begin{array}{l}\$ 2.11(1.82 \\
2.41)\end{array}$ & $\begin{array}{l}\$ 3.64(3.43 \\
3.86)\end{array}$ & $\begin{array}{l}\$ 18.54(15.04 \\
22.36)^{\mathrm{a}}\end{array}$ & $\begin{array}{l}\$ 14.12(12.98 \\
15.30)^{b}\end{array}$ & $\$ 4.99(4.53,5.46)^{c}$ & $\$ 16.98(13.94,20.45)^{d}$ \\
\hline Diagnostics & $\begin{array}{l}\$ 9.24(8.30 \\
10.35)\end{array}$ & $\begin{array}{l}\$ 3.64(3.34, \\
3.97)\end{array}$ & $\begin{array}{l}\$ 0.45(0.32, \\
0.61)\end{array}$ & $\begin{array}{l}\$ 9.65(7.31 \\
11.99)\end{array}$ & $\begin{array}{l}\$ 6.81(6.08 \\
7.54)\end{array}$ & $\$ 3.87(3.49,4.23)$ & $\$ 9.07(7.08,11.08)$ \\
\hline Overheads & $\begin{array}{l}\$ 5.39(5.16 \\
5.63)\end{array}$ & $\begin{array}{l}\$ 5.19(4.80 \\
5.59)\end{array}$ & $\begin{array}{l}\$ 6.36(6.04 \\
6.67)\end{array}$ & $\begin{array}{l}\$ 4.21(3.61 \\
4.86)\end{array}$ & $\begin{array}{l}\$ 4.64(4.33, \\
4.95)\end{array}$ & $\$ 6.07(5.72,6.42)$ & $\$ 3.97(3.39,4.60)$ \\
\hline Administration & $\begin{array}{l}\$ 3.61(3.39, \\
3.82)\end{array}$ & $\begin{array}{l}\$ 3.69(3.36 \\
4.03)\end{array}$ & $\begin{array}{l}\$ 4.56(4.27 \\
4.85)\end{array}$ & $\begin{array}{l}\$ 2.43(2.03, \\
2.95)\end{array}$ & $\begin{array}{l}\$ 2.78(2.52, \\
3.06)\end{array}$ & $\$ 4.19(3.89,4.49)$ & $\$ 2.25(1.87,2.73)$ \\
\hline $\begin{array}{l}\text { Equipment and } \\
\text { furniture }\end{array}$ & $\begin{array}{l}\$ 0.38(0.35 \\
0.41)\end{array}$ & $\begin{array}{l}\$ 0.34(0.29 \\
0.38)\end{array}$ & $\begin{array}{l}\$ 0.40(0.36 \\
0.43)\end{array}$ & $\begin{array}{l}\$ 0.44(0.31 \\
0.56)\end{array}$ & $\begin{array}{l}\$ 0.39(0.34 \\
0.44)\end{array}$ & $\$ 0.51(0.46,0.56)$ & $\$ 0.38(0.27,0.50)$ \\
\hline Rental space & $\begin{array}{l}\$ 1.41(1.37 \\
1.44)\end{array}$ & $\begin{array}{l}\$ 1.16(1.11 \\
1.22)\end{array}$ & $\begin{array}{l}\$ 1.40(1.36 \\
1.43)\end{array}$ & $\begin{array}{l}\$ 1.34(1.16 \\
1.51)\end{array}$ & $\begin{array}{l}\$ 1.47(1.40 \\
1.53)\end{array}$ & $\$ 1.37(1.32,1.42)$ & $\$ 1.34(1.16,1.51)$ \\
\hline
\end{tabular}

${ }^{a}$ The mean cost for antiretroviral therapy was $\$ 16.92(95 \% \mathrm{Cl} 13.22,20.84)$ and that of diabetes drugs was $\$ 1.62(95 \% \mathrm{Cl} 0.96,2.39)$

${ }^{\mathrm{b}}$ The mean cost for antiretroviral therapy was $\$ 11.88(95 \% \mathrm{Cl} 10.81,12.99)$ and that of hypertension drugs was $\$ 2.24(95 \% \mathrm{Cl} 1.91,2.59)$

'The mean cost of diabetes medication was $\$ 1.75(95 \% \mathrm{Cl} 1.44,2.09)$ and that of hypertension medication was $\$ 3.24(95 \% \mathrm{Cl} 2.95,3.54)$

${ }^{\mathrm{d}}$ The mean cost for antiretroviral therapy was $\$ 13.26(95 \% \mathrm{Cl} 10.62,16.15)$, hypertension drugs was $\$ 2.53(95 \% \mathrm{Cl} 1.78,3.35)$ and that of diabetes drugs was $\$ 1.19$ $(95 \% \mathrm{Cl} 0.64,1.94)$

${ }^{1}$ The cost of managing an individual with HIV and diabetes was $-\$ 13.39(95 \% \mathrm{Cl}-18.69,-8.02)$ cheaper than managing two individuals with single conditions of HIV and diabetes, i.e., cost reductions of $25.5 \%(95 \% \mathrm{Cl} 15.4 \%, 35.5 \%)$

${ }^{2}$ The cost of managing an individual with HIV and hypertension was - $\$ 20.63(95 \% \mathrm{Cl}-23.00,-18.31)$ cheaper than managing two individuals with single conditions of HIV and hypertension, i.e., cost reductions of $39.1 \%(95 \% \mathrm{Cl} 35.1 \%, 42.9 \%)$

${ }^{3}$ The cost of managing an individual with diabetes and hypertension was - $\$ 14.24(95 \% \mathrm{Cl}-15.42,-13.05)$ cheaper than managing two individuals with single conditions of diabetes and hypertension, i.e., cost reduction of $38.6 \%(95 \% \mathrm{Cl} 36.0 \%, 41.1 \%)$

${ }^{4}$ The cost of managing an individual with three conditions HIV, diabetes and hypertension is $-\$ 34.72(95 \% \mathrm{Cl}-39.53,-29.75) \mathrm{cheaper}$ than the cost of managing three individuals with single conditions of HIV, diabetes and hypertension, i.e., cost reductions of $48.8 \%$ (95\% $\mathrm{Cl} 42.1 \%, 55.3 \%)$. The overall cost of managing individuals with multi-morbidity ( 2 or 3 conditions) is $-\$ 20.74(95 \% \mathrm{Cl}-37.94,-9.83$ ) cheaper than the cost of two or more individuals with single conditions, i.e., cost reductions of $38.0 \%$ (95\% Cl $18.8 \%, 53.1 \%)$

\#The average additional cost per month of managing: (i) diabetes in an HIV-infected individual is $\$ 4.89$ (95\% Cl - 0.36, 10.22), (ii) hypertension in HIV-infected individual is $-\$ 2.03(95 \% \mathrm{Cl}-4.33,0.27)$ and (iii) hypertension in diabetic individuals is $\$ 4.37(95 \% \mathrm{Cl} 3.27,5.49)$

was respectively $17.8 \%(9.3,27.5), 11.7 \%(8.9,14.8)$ and $12.2 \%(10.0,14.6)$. Hence, the latter are the groups that benefit most from one-stop clinics and become less financially challenged.

\section{Benchmark efficiency of service provision among integrated health facilities}

To assess the efficiency changes of service delivery through the integration of services, we used the staff numbers, the number of diagnostic tests and drug availability collected at each facility (grouped as HIV clinics, NCD clinics and outpatient departments) as key quality indicators for each facility performance. We show that there was a varying level of efficiency in the service delivery among some sites (Table 6), as the mean efficiency score of a scale from 0.0 to 1.0 was 0.86 (range for all 10 facilities, $0.30-1.00$ ). This suggests that some facilities had room to add additional participants without the need to increase the key staff numbers and without compromising their current standard or quality of care. So some HIV clinics and outpatient departments had capacity to accommodate extra participants without extra staff costs, whereas none of NCD clinics had such extra capacity (Table 6). We included the overcapacity estimates based on the DEA to adjust the prevalence-based budget projections downwards.

\section{Discussion}

This detailed socio-economic study shows that integrated one-stop management of chronic conditions specifically HIV infection, diabetes and hypertension could reduce costs per participant between 34 to almost $50 \%$, compared to managing each condition separately and hence is a highly cost-saving approach in African settings. The savings are mostly among people presenting with multiple conditions, who are financially most affected. Integration lowered the mean health service cost per visit through a one-time use of both personnel and capital resources. Participants with multiple conditions made one trip every 1 to 3 months to a health facility under the integrated care model. This is more convenient and less costly than two or three visits as is the case across sub-Saharan Africa now, if participants are treated in vertical stand-alone clinicals for each 
Table 4 Income and health cost for participants with single, two and three conditions attending an integrated one-stop clinic in Uganda and Tanzania. The mean costs and 95\% confidence intervals reported are in 2020 US\$

\begin{tabular}{|c|c|c|c|c|c|c|c|}
\hline Cost component & HIV only & Diabetes & Hypertension & $\begin{array}{l}\text { HIV + } \\
\text { diabetes }\end{array}$ & $\begin{array}{l}\text { HIV + } \\
\text { hypertension }\end{array}$ & $\begin{array}{l}\text { Diabetes + } \\
\text { hypertension }\end{array}$ & $\begin{array}{l}\text { HIV + diabetes + } \\
\text { hypertension }\end{array}$ \\
\hline Consultation costs & $\begin{array}{l}\$ 0.20(0.12 \\
0.30)\end{array}$ & $\begin{array}{l}\$ 0.81(0.45, \\
1.27)\end{array}$ & $\begin{array}{l}\$ 0.27(0.13 \\
0.44)\end{array}$ & $\begin{array}{l}\$ 0.68(0.00 \\
1.58)\end{array}$ & $\begin{array}{l}\$ 0.08(0.00 \\
0.19)\end{array}$ & $\begin{array}{l}\$ 0.50(0.26 \\
0.77)\end{array}$ & $\$ 0.16(0.00,0.46)$ \\
\hline Treatment costs & $\begin{array}{l}\$ 0.05(0.00, \\
0.12)\end{array}$ & $\begin{array}{l}\$ 2.64(0.96, \\
5.26)\end{array}$ & $\begin{array}{l}\$ 0.67(0.15 \\
1.53)\end{array}$ & $\begin{array}{l}\$ 1.01(0.00, \\
2.72)\end{array}$ & $\begin{array}{l}\$ 0.01(0.00, \\
0.04)\end{array}$ & $\begin{array}{l}\$ 0.96(0.21 \\
2.21)\end{array}$ & $\$ 0.00(0.00,0.00)$ \\
\hline Medication costs & $\begin{array}{l}\$ 0.03(0.00 \\
0.08)\end{array}$ & $\begin{array}{l}\$ 4.81(2.49, \\
7.53)\end{array}$ & $\begin{array}{l}\$ 1.69(0.81 \\
2.80)\end{array}$ & $\begin{array}{l}\$ 4.40(0.00 \\
10.58)\end{array}$ & $\begin{array}{l}\$ 0.14(0.00, \\
0.38)\end{array}$ & $\begin{array}{l}\$ 3.78(1.30 \\
7.25)\end{array}$ & $\$ 0.90(0.00,2.45)$ \\
\hline Transport costs & $\begin{array}{l}\$ 1.37(1.24, \\
1.52)\end{array}$ & $\begin{array}{l}\$ 1.16(0.96, \\
1.39)\end{array}$ & $\begin{array}{l}\$ 0.86(0.75 \\
0.97)\end{array}$ & $\begin{array}{l}\$ 1.85(1.29, \\
2.43)\end{array}$ & $\begin{array}{l}\$ 1.66(1.33, \\
2.03)\end{array}$ & $\begin{array}{l}\$ 1.46(1.17 \\
1.79)\end{array}$ & $\$ 1.73(1.28,2.22)$ \\
\hline Transport costs for other persons & $\begin{array}{l}\$ 0.07(0.04 \\
0.10)\end{array}$ & $\begin{array}{l}\$ 0.15(0.07, \\
0.24)\end{array}$ & $\begin{array}{l}\$ 0.09(0.06 \\
0.13)\end{array}$ & $\begin{array}{l}\$ 0.05(0.00 \\
0.14)\end{array}$ & $\begin{array}{l}\$ 0.10(0.03 \\
0.20)\end{array}$ & $\begin{array}{l}\$ 0.22(0.07 \\
0.42)\end{array}$ & $\$ 0.12(0.00,0.30)$ \\
\hline Lost labour costs & $\begin{array}{l}\$ 0.92(0.73 \\
1.13)\end{array}$ & $\begin{array}{l}\$ 0.90(0.66, \\
1.18)\end{array}$ & $\begin{array}{l}\$ 0.50(0.38, \\
0.67)\end{array}$ & $\begin{array}{l}\$ 0.70(0.33 \\
1.19)\end{array}$ & $\begin{array}{l}\$ 0.82(0.59, \\
1.08)\end{array}$ & $\begin{array}{l}\$ 0.74(0.50 \\
1.06)\end{array}$ & $\$ 0.95(0.39,1.83)$ \\
\hline Other costs (food) & $\begin{array}{l}\$ 1.56(1.39 \\
1.76)\end{array}$ & $\begin{array}{l}\$ 1.85(1.53, \\
2.21)\end{array}$ & $\begin{array}{l}\$ 1.57(1.32 \\
1.88)\end{array}$ & $\begin{array}{l}\$ 1.48(0.82 \\
2.35)\end{array}$ & $\begin{array}{l}\$ 1.30(1.03 \\
1.63)\end{array}$ & $\begin{array}{l}\$ 2.02(1.53, \\
2.63)\end{array}$ & $\$ 1.34(0.95,1.80)$ \\
\hline Total monthly patient-related costs ${ }^{a}$ & $\begin{array}{l}\$ 4.20(3.82, \\
4.61)\end{array}$ & $\begin{array}{l}\$ 12.32 \\
(8.13 \\
17.48)\end{array}$ & $\begin{array}{l}\$ 5.66(4.15 \\
7.61)\end{array}$ & $\begin{array}{l}\$ 10.17 \\
(3.79 \\
18.75)\end{array}$ & $\begin{array}{l}\$ 4.11(3.47, \\
4.84)\end{array}$ & $\begin{array}{l}\$ 9.68(6.10 \\
14.78)\end{array}$ & $\$ 5.20(3.71,7.02)$ \\
\hline Average monthly household income & $\begin{array}{l}\$ 75.15 \\
(65.34, \\
86.95)\end{array}$ & $\begin{array}{l}\$ 125.4 \\
(73.55, \\
214.9)\end{array}$ & $\begin{array}{l}\$ 89.43(70.86 \\
111.2)\end{array}$ & $\begin{array}{l}\$ 81.81 \\
(52.26 \\
115.2)\end{array}$ & $\begin{array}{l}\$ 79.09(59.68 \\
101.3)\end{array}$ & $\begin{array}{l}\$ 95.20(67.41 \\
136.0)\end{array}$ & $\begin{array}{l}\$ 76.23(42.66 \\
117.0)\end{array}$ \\
\hline Total integrated costs ${ }^{\mathrm{b}}$ & $\begin{array}{l}\$ 38.38 \\
(37.03 \\
39.83)\end{array}$ & $\begin{array}{l}\$ 25.80 \\
(23.42 \\
28.88)\end{array}$ & $\begin{array}{l}\$ 22.57(21.65 \\
23.68)\end{array}$ & $\begin{array}{l}\$ 44.88 \\
(39.17 \\
50.81)\end{array}$ & $\begin{array}{l}\$ 36.15(34.20, \\
38.11)\end{array}$ & $\begin{array}{l}\$ 28.55(26.93 \\
30.61)\end{array}$ & $\begin{array}{l}\$ 40.67(36.04 \\
45.44)\end{array}$ \\
\hline $\begin{array}{l}\% \text { of transport costs as a function of } \\
\text { monthly household income }\end{array}$ & $\begin{array}{l}4.4 \%(3.7, \\
5.2)\end{array}$ & $\begin{array}{l}3.0 \%(2.1 \\
4.0)\end{array}$ & $2.5 \%(2.0,3.2)$ & $\begin{array}{l}6.9 \%(2.4 \\
13.2)\end{array}$ & $6.2 \%(4.3,8.6)$ & $4.4 \%(3.2,5.8)$ & $3.8 \%(2.1,5.9)$ \\
\hline $\begin{array}{l}\% \text { of total patient-related costs as a } \\
\text { function of monthly income }\end{array}$ & $\begin{array}{l}9.3 \%(8.3 \\
10.5)\end{array}$ & $\begin{array}{l}12.2 \%(9.7 \\
14.8)\end{array}$ & $\begin{array}{l}9.2 \%(7.5 \\
11.1)\end{array}$ & $\begin{array}{l}17.8 \%(9.3 \\
27.5)\end{array}$ & $\begin{array}{l}11.7 \%(8.9, \\
14.8)\end{array}$ & $\begin{array}{l}12.2 \%(10.0 \\
14.6)\end{array}$ & $9.3 \%(5.8,13.4)$ \\
\hline $\begin{array}{l}\% \text { of total patient-related costs as a } \\
\text { function of total integrated cost } \mathrm{e}^{\mathrm{e}}\end{array}$ & $\begin{array}{l}10.9 \% \\
(10.0,12.1)\end{array}$ & $\begin{array}{l}47.5 \% \\
(34.0,61.8)\end{array}$ & $\begin{array}{l}25.0 \%(18.9 \\
32.4)\end{array}$ & $\begin{array}{l}22.5 \%(8.9 \\
39.9)\end{array}$ & $\begin{array}{l}11.4 \%(9.7 \\
13.3)\end{array}$ & $\begin{array}{l}33.7 \%(22.3 \\
48.8)\end{array}$ & $12.8 \%(9.2,17.4)$ \\
\hline
\end{tabular}

${ }^{a}$ Average patient costs for all participants combined is $\$ 7.33(95 \% \mathrm{Cl} 3.70,15.86)$ with transport and medication costs of $\$ 1.44(95 \% \mathrm{Cl} 0.81,2.18)$ and $\$ 2.25(95 \%$ $\mathrm{Cl} 0.00,7.55)$, respectively

${ }^{\mathrm{b}}$ Total integrated cost is the sum of total health service cost (Table 3) and total patient-related costs minus patient-paid medication costs to avoid duplication 'The proportion of transport cost as a function of the monthly household income per health care visit for patients with single conditions was $3.3 \%$ ( $95 \%$ Cl 2.1 , $4.9)$ and $5.3 \%(95 \% \mathrm{Cl} 2.4,10.7)$ for patients with multiple conditions. The overall proportion of transport costs as a function of monthly household income was $4.5 \%(95 \% \mathrm{Cl} 2.1,9.6)$. Computed only for participants with a household income

${ }^{\mathrm{d}}$ The proportion of total out-of-pocket expenditure as a function of the monthly household income per health care visit for patients with single conditions was $10.3 \%(95 \% \mathrm{Cl} 7.9,14.2)$ and $12.7 \%(95 \% \mathrm{Cl} 6.8,24.0)$ for patients with multiple conditions. The overall proportion of patient-related costs as a function of monthly household income was $11.7 \%(95 \% \mathrm{Cl} 7.3,22.1)$. Computed only for participants with a household income

'The overall proportion of total patient-related costs as a function of total integrated cost was $23.4 \%(95 \% \mathrm{Cl} 9.9,54.3)$

condition. This results in huge out-of-pocket savings for them and reduced duplication of clinical management activities at the health service.

At the health service, the added cost of managing multimorbidity is essentially determined by the additional costs of drugs and diagnostics for the second or third chronic condition. Savings are realised through reductions in health worker time and in patients' time, per participant. The time taken by health care workers, including doctors and nurses, appeared to be similar whether they were managing one condition or all three. This is not uncommon in clinical practice. It might be possible that clinical staff, working under resource constraints, allocate a fixed amount of time per participant and so did not need to give more time or attention to the multiple conditions in the absence of specific complications. Our clinical findings on a one-year follow-up [12] suggest that people with multimorbidity had better outcomes in terms of HIV viral suppression, glycaemia and blood pressure control than people with single diseases. It is more likely, that key elements of the management of chronic conditions - clinical monitoring for disease progression and promoting drug adherence - are similar for these chronic diseases and one clinical consultation more effectively covers all of these conditions, allowing consultation time to be saved, more patients to be served, and better quality care to be delivered as indicated by the better clinical outcomes [12].

A major challenge in the management of diabetes and hypertension in Africa has been the limited and erratic supply of drugs and diagnostics [28]. Our study shows 
Table 5 Health worker and patient cost by disease condition based on measured time involvement (US\$2020). Mean costs and 95\% confidence intervals are given in parentheses

\begin{tabular}{|c|c|c|c|c|c|c|c|}
\hline Provider & HIV only & Diabetes & Hypertension & $\begin{array}{l}\text { HIV + } \\
\text { diabetes }\end{array}$ & $\begin{array}{l}\mathrm{HIV}+ \\
\text { hypertension }\end{array}$ & $\begin{array}{l}\text { Diabetes + } \\
\text { hypertension }\end{array}$ & $\begin{array}{l}\text { HIV + diabetes + } \\
\text { hypertension }\end{array}$ \\
\hline Clinician & $\begin{array}{l}\$ 1.70(1.49, \\
2.08)\end{array}$ & $\begin{array}{l}\$ 1.96(1.49, \\
2.37)\end{array}$ & $\begin{array}{l}\$ 1.59(1.32, \\
2.00)\end{array}$ & $\begin{array}{l}\$ 1.92(1.68, \\
2.18)\end{array}$ & $\$ 1.82(1.41,2.59)$ & $\$ 1.45(1.16,1.69)$ & $\$ 2.25(1.52,2.81)$ \\
\hline Technician & $\begin{array}{l}\$ 0.43(0.33 \\
0.60)\end{array}$ & $\begin{array}{l}\$ 0.79(0.55 \\
1.16)\end{array}$ & $\begin{array}{l}\$ 0.41(0.20, \\
0.61)\end{array}$ & $\begin{array}{l}\$ 0.60(0.54 \\
0.71)\end{array}$ & $\$ 0.76(0.55,0.93)$ & $\$ 0.59(0.43,0.96)$ & $\$ 1.05(0.55,1.61)$ \\
\hline Nurse & $\begin{array}{l}\$ 0.54(0.35 \\
0.71)\end{array}$ & $\begin{array}{l}\$ 0.56(0.36 \\
0.74)\end{array}$ & $\begin{array}{l}\$ 0.44(0.26 \\
0.65)\end{array}$ & $\begin{array}{l}\$ 0.48(0.36 \\
0.55)\end{array}$ & $\$ 0.99(0.49,1.58)$ & $\$ 0.32(0.20,0.45)$ & $\$ 0.49(0.42,0.56)$ \\
\hline Pharmacist & $\begin{array}{l}\$ 0.34(0.23 \\
0.43)\end{array}$ & $\begin{array}{l}\$ 0.58(0.30 \\
0.78)\end{array}$ & $\begin{array}{l}\$ 0.50(0.41 \\
0.63)\end{array}$ & $\begin{array}{l}\$ 0.37(0.15 \\
0.76)\end{array}$ & $\$ 0.49(0.27,0.99)$ & $\$ 0.34(0.15,0.51)$ & $\$ 0.16(0.12,0.21)$ \\
\hline Triage & $\begin{array}{l}\$ 0.93(0.56 \\
1.37)\end{array}$ & $\begin{array}{l}\$ 0.96(0.62 \\
1.44)\end{array}$ & $\begin{array}{l}\$ 0.71(0.47 \\
0.90)\end{array}$ & $\begin{array}{l}\$ 0.41(0.18 \\
0.61)\end{array}$ & $\$ 0.92(0.35,2.35)$ & $\$ 0.50(0.33,0.75)$ & $\$ 1.72(0.30,2.60)$ \\
\hline Patient & $\begin{array}{l}\$ 1.42(0.85 \\
2.42)\end{array}$ & $\begin{array}{l}\$ 1.26(1.13 \\
1.47)\end{array}$ & $\begin{array}{l}\$ 1.86(1.35, \\
2.91)\end{array}$ & $\begin{array}{l}\$ 1.44(0.95 \\
2.13)\end{array}$ & $\$ 1.05(0.67,1.37)$ & $\$ 1.57(1.17,2.42)$ & $\$ 1.59(0.99,2.63)$ \\
\hline
\end{tabular}

that the costs for both conditions combined are still lower than the costs of managing HIV infection. Our HIV costs were like those derived in an earlier study [29]. In the prevalence-based budget projection for the year 2025, we considered demographic changes as well disease-specific frailty in surviving all participant categories. It shows savings of $21.6 \%$ from the integrated management of the three conditions included (see annex). Given that far more people in Africa today die from the complications associated with diabetes and hypertension than with HIV infection, it is essential that that integrated care for multi-morbidities within country health systems is strengthened. Even then, transport and other related costs will still be high and may even be crippling for some households, as our study shows. New innovative ways of managing chronic conditions are needed, such as community care, inclusion of e-health and less frequent appointments at health facilities, as is the case for HIV infections.

In high-income countries, about $25 \%$ of adults have multi-morbidity from chronic conditions [30,31], while the economic burden of multi-morbidity is largely unknown. The exact burden is unknown in Africa, but non-communicable conditions have risen sharply [32, 33] alongside a continuing high burden of HIV infection and new models of chronic care management will be needed in sub-Sahara Africa. Based on existing population projections and the empirical health service costs derived in this study, we estimate the economic health service burden in people living with comorbidity (HIV and/or diabetes and/or hypertension) will go up by $21.5 \%$ (range, $19.3 \%, 23.4 \%$ ) on average, for the next 5 years, despite the included savings of $21.6 \%$ from the integration of services. By year 2030, the economic burden may increase by $47.7 \%$ (range, $41.9 \%, 52.7 \%$ ) compared to the current economic burden in year 2020 (Fig. 2 and Table A7 in the Additional file 1 and country statistics). Even with the ongoing changing epidemiology, most people in Africa will likely have a single chronic condition, at least for the decade to come and the costs associated with this in a vertical clinical, compared with our model, are unlikely to be appreciably different. The key

Table 6 Relative facility efficiency scores on the delivery of integrated care for people with HIV, diabetes and/or hypertension. Values are given as means and ranges in parentheses

\begin{tabular}{|c|c|c|c|c|c|c|c|}
\hline \multirow[t]{3}{*}{ Clinic } & \multirow{2}{*}{\multicolumn{3}{|c|}{$\frac{\text { Inputs }}{\text { Staff }}$}} & \multicolumn{3}{|l|}{ Outputs } & \multirow{3}{*}{$\begin{array}{l}\text { Relative } \\
\text { Efficiency }\end{array}$} \\
\hline & & & & \multirow{2}{*}{$\begin{array}{l}\text { Drug } \\
\text { availability }^{\mathrm{a}}\end{array}$} & \multirow{2}{*}{$\begin{array}{l}\text { Staff } \\
\text { workload (per } \\
1000 \text { patient } \\
\text { visits) }^{b}\end{array}$} & \multirow{2}{*}{$\begin{array}{l}\text { Number of } \\
\text { laboratory } \\
\text { tests per } \\
\text { patient }^{c}\end{array}$} & \\
\hline & Doctors & Nurses & Other staff & & & & \\
\hline Overall & $3(0-8)$ & $4(1-11)$ & $6(2-16)$ & $0.82(0.01-1.00)$ & $0.39(0.13-1.36)$ & $0.61(0.00-1.81)$ & $0.86(0.30-1.00)$ \\
\hline HIV & $3(2-5)$ & $3(2-6)$ & $4(2-6)$ & $1.00(1.00-1.00)$ & $0.30(0.23-0.44)$ & $0.91(0.25-1.81)$ & $0.89(0.35-1.00)$ \\
\hline NCD & $1(0-3)$ & $2(1-5)$ & $6(2-14)$ & $0.68(0.40-1.00)$ & $0.48(0.19-1.11)$ & $0.30(0.09-0.63)$ & $1.00(1.00-1.00)$ \\
\hline OPD & $5(3-8)$ & $7(4-11)$ & $9(3-16)$ & $0.60(0.01-1.00)$ & $0.47(0.13-1.36)$ & $0.33(0.00-1.47)$ & $0.68(0.30-1.00)$ \\
\hline
\end{tabular}

NCD non-communicable disease, OPD outpatient department Other staff: this includes laboratory personnel and pharmacists

${ }^{a}$ Drug availability - defined as the proportion of patients who get free drugs from the facilities before stock-outs occur

${ }^{\text {b }}$ Staff workload - the number of full-time staff divided by the number of daily patient visits

${ }^{\mathrm{c}}$ Number of laboratory tests per patient - the number of laboratory tests divided by the number of patients' visits 

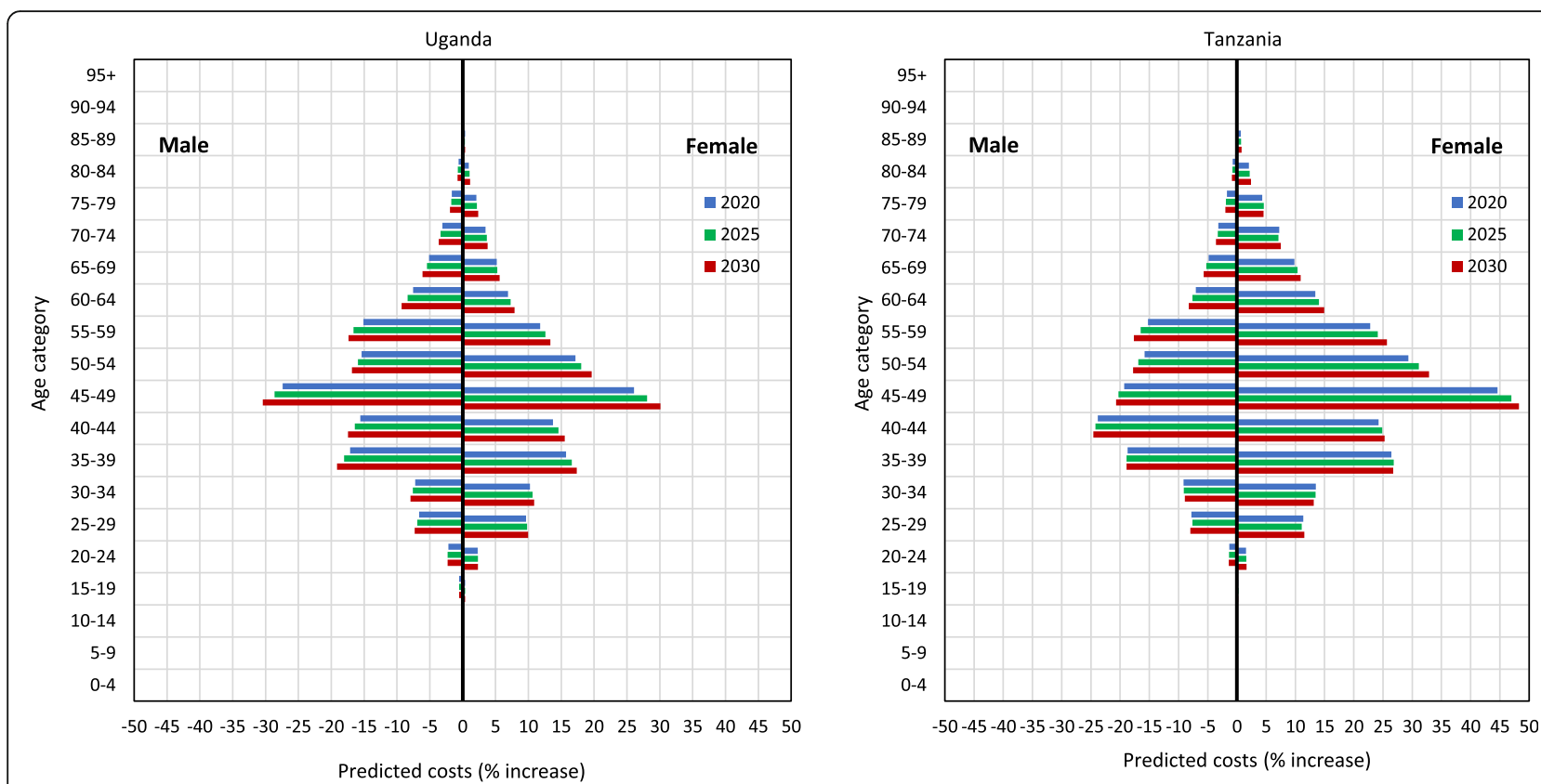

Fig. 2 The effect of ageing and decreased survival on the total health service costs of managing people living with multiple conditions (HIV and/ or diabetes and/or hypertension) in Uganda and Tanzania in years 2020, 2025 and 2030, compared to baseline

question will be which models of care lead to better clinical and economic outcomes for patients with multiple conditions.

This first economic cluster-based study on real-life integrated care has some study design limitations to consider because it comprises a single-arm cohort over a 1year follow-up duration [15, 34]. In the explorative phase of developing integrated care, we set-up this cohort without a control arm to test its acceptability to patients. Here, patients were given a choice to participate, as HIV control in a new integrated setting could be weakened due to stigma. This has guided the choice for a cohort design that has demonstrated an increase in compliance and control levels [12]. In our cohort study, a comparison group in a real-life setting (e.g., in a before-after study or a control arm) would not present the correct comparison in the absence of consistent drug supplies, as in real-life shortages in diagnostics and medicines for diabetes and hypertension control are common and patient adherence is therefore, likely, very limited. Secondly, while one may consider it unethical to withhold proven-effective treatment in control groups [35], our next follow-up study - already started - is a clusterrandomised trial based, therefore, on equipoise effects and harm of integration, pursuing a more rigorous and larger design that is needed to assess the effects of integration $[15,36]$.

We want to argue that real-life economic costing studies are less subject to Hawthorne effects among providers or patient participants and that the clusters are less prone to contamination: the benefits are less tangible, and hence, the estimates are less biassed. We did compare large groups of participants with a single condition to large within-cohort groups of participants with multiple conditions in both countries and consider the approach, in principle, rather robust, given the study design. Also, the follow-up period is long enough to arrive stable economic estimates of clinical resource use as patients are already diagnosed and the treatment of patients with chronic conditions tend to stabilise within six months, especially those with multiple conditions [12].

In principle, we expect some generalizable mechanisms across similar settings as the potential service effects (less programme costs per patient and less health worker cost) and effects for patients (reduction of visits) are both rather intuitive, certainly for people with multiple conditions. This will need to be proven in a larger multi-country cluster-trial, considering the existing situation and the implementation costs of the upscaled efforts. In future research, one must distinguish carefully studies addressing multi-morbidities and those addressing integration of care. The former would need individual randomisation while the latter needs clusterrandomisation at a large scale.

However, presently, one cannot say much about country differences. We need more qualitative work [14] and larger samples in the quantitative research, including step-wedge designs.

There are many factors that will influence how integrated services are implemented or the potential impact 
that the adoption of integration will have. As this was a feasibility study, the findings are indicative of impact, but more robust evidence is required before definitive recommendations can be made. Impact will be influenced by the prevalence of the individual conditions, organisation of health services including availability of insurance and policy directions in each country. Based on experience since the end of MOCCA, it seems that integration might be better suited to smaller health facilities with more limited resources. Larger health facilities that are delivering more specialised care or who are accepting referrals may be better suited to only implementing some aspects of integration. In Uganda, the smaller health facilities in the study have since fully integrated their services after the end of MOCCA. This has increased the provision of services, i.e., where there were two HIV clinics per week, this is now available every day. This is explored further in the MOCCA extension study. In general, in other settings, one can expect between-facility differences at baseline. Integration would probably be less impactful in small facilities where patients with different conditions are already managed through the same channels, providing one other clear mechanism towards integrated care. Ultimately, policy decisions about integrated clinics need to combine general principles with local factors.

\section{Conclusion}

In sum, this study is a first valid exploratory approach to detail the economic costs and economies-of-scale of integrating services for chronic conditions in integrated one-stop clinics in sub-Sahara Africa. It provides much needed evidence on the probable efficiency of such models of service provision for HIV, diabetes and hypertension. Larger studies comparing directly, possibly through multiple arms directly integrated primary care with more vertical care models in a facility-randomised way will be useful to assess the overall marginal consequences, in terms of service and household impact of integrated care. The first results are certainly very promising.

In this cohort study, we have demonstrated that onestop treatment of comorbidities is likely worthwhile and an efficient strategy enhancing financial equity in service provision for people with multiple conditions in subSaharan Africa settings.

\section{Supplementary Information}

The online version contains supplementary material available at https://doi. org/10.1186/s12916-021-02094-2.

Additional file 1: Table A1. Unit prices (2019/2020 Ugandan and

Tanzanian Shillings). Table A2. Resource use specifications by single,

double, and triple disease. Table A3. Monthly programme healthcare costs (US\$ 2019/20) per patient by disease and facility. This includes staff salaries including non-clinical staff such as cleaners and drivers, drugs, medical consumables and supplies, laboratory testing, medical equipment, physical infrastructure used for patient care. Table A4. Out-ofpocket expenses and loss of earnings (median and ranges) in 2010/2020 US\$. Table A5. Projected costs of managing people living with HIV, diabetes, hypertension, and multi-morbidity by gender in Tanzania and Uganda. Table A8. Prevalence of diabetes in Tanzania and Uganda [1]. Table A9. Prevalence of hypertension in Tanzania and Uganda [2, 3]. Table A10. HIV prevalence by age and gender in Tanzania and Uganda. Table A11. Projected impact of aging and differences in survival on the number of people living with HIV, diabetes, hypertension, and multimorbidity (in thousands) by gender in Tanzania and Uganda. Sample size for the time and motion observational study. Figure A1. The effect of aging on the increase in the number of people with combined conditions (HIV and/or diabetes and/or hypertension) compared to cases in year 2020 by gender in Uganda and Tanzania.

\section{Acknowledgements}

The one-stop clinic model, as a novel approach, needed substantial stakeholder engagement. In each country, we held planning meetings with the Ministry of Health and non-governmental partners at national and local level, with senior clinicians and hospital managers and with patient and community representatives. National steering committees were formed, comprising representatives from these stakeholders together with the researchers. Patient representatives attended our planning meetings and contributed to the design of the study and other aspects of the research, such as its implementation. We set up steering committees in both Tanzania and Uganda, which comprised researchers, policy makers and had patient representatives to oversee the implementation. We also held investigator meetings involving all the partners. These included patient representatives. We want to acknowledge all, here.

Next, we would like to thank the patient participants and health workers for their participation. We also acknowledge the collaborations within the RESPOND consortium among the study teams in Uganda, Tanzania and Liverpool, in particular our colleagues for their help with data management and their contributions to the discussions on this sub-study: Ayubu Masasi, Ayoub Kakande, Luis Cuevas, Bernard Etukoit, Geoff Gill, Janet Lutale, Levi Mugenyi, Kenneth Mugisha, Hazel Snell, Duolao Wang and Erik van Widenfelt.

\section{Authors' contributions}

LN, SJ, MJN, SM, AVG, SLK, JB, GM, JMg, JMu, JL and NKS conceptualised and designed the present study with support from KR. TS, SLK, JB, IN and GK implemented the study and analysed the data, with support from LN, SJ, AVG, SM and JMu. TS, SLK, JB and IN managed the data with support from AVG, GK and JO while TS oversaw the collection of unit prices. TS wrote the first drafts and LN and SJ led the later drafts. TS did the statistical analysis with support from LN. All authors contributed to interpretation of data and substantially revised subsequent drafts of the manuscript. The authors read and approved the final version.

\section{Funding}

This research was funded by the National Institute for Health Research (NIHR) (project reference 16/137/87) using UK aid from the UK Government to support global health research. The views expressed in this publication are those of the authors and not necessarily those of the NIHR or the UK Department of Health and Social Care.

\section{Availability of data and materials}

There is an annex to the manuscript that gives details of the economic study including unit prices and the details of budget projection and data sources. Underlying data on the presented findings are available from the authors.

\section{Declarations}

Ethics approval and consent to participate

The study was approved by the Research Ethics Committee of the Liverpool School of Tropical Medicine (LSTM REC 18-044), the Research Ethics Committee of The AIDS Support Organisation (TASO/REC/015/18-UG-REC-009) in 
Uganda, and the National Health Research Ethics Review Committee with its secretariat at the National Institute for Medical Research (NIMR/HQ/R.8a/Nol. IX/2793) in Tanzania. The trial was done according to the Declaration of Helsinki and in accordance with Good Clinical Practice guidelines. At each clinic, the research team employed one data clerk and one research nurse to inform patients about the research, collect research data and take informed consent. All included patients signed an informed consent form. Small amounts of salary (salary top-ups) were provided to selected facility staff working with study participants and to the head nurse responsible for operations to ensure accurate data collection. Research staff worked alongside the health facility staff.

\section{Consent for publication}

All authors have seen the final version of the manuscript and agree with publication.

\section{Competing interests}

The authors declare that they have no competing interests.

\section{Author details}

${ }^{1}$ Liverpool School of Tropical Medicine, Pembroke Place, Liverpool L3 5QA, UK. ${ }^{2}$ The AIDS Support Organisation, Mulago Hospital Complex, Kampala, Uganda. ${ }^{3}$ Medical Research Council/Uganda Virus Research Institute \& London School of Hygiene and Tropical Medicine Uganda Research Unit, Entebbe, Uganda. ${ }^{4}$ National Institutes for Medical Research, Dar es Salaam, Tanzania. ${ }^{5}$ Directors Office, Ministry of Health, Community Development, Gender, Elderly and Children, Kampala, Uganda. ${ }^{6}$ School of Public Health, Muhimbili University of Health and Allied Sciences, Dar es Salaam, Tanzania. ${ }^{7}$ Non-Communicable Diseases Control Programme, Ministry of Health, Kampala, Uganda. ${ }^{8}$ London School of Hygiene \& Tropical Medicine, Keppel Street, London WC1E 7HT, UK. ${ }^{9}$ Hindu Mandal Hospital, Dar es Salaam, Tanzania. ${ }^{10}$ Norwich Medical School, University of East Anglia, Norwich, UK. ${ }^{11}$ Makerere University College of Health Sciences, Kampala, Uganda. ${ }^{12}$ Department of International Health, Johns Hopkins Bloomberg School of Public Health, Baltimore, MD, USA.

\section{Received: 15 February 2021 Accepted: 11 August 2021} Published online: 10 September 2021

\section{References}

1. Atun R, Davies JI, Gale EAM, Bärnighausen T, Beran D, Kengne AP, et al. Diabetes in sub-Saharan Africa: from clinical care to health policy. Lancet Diabetes Endocrinol. 2017;5(8):622-67. https://doi.org/10.1016/S2213-8587(1 7)30181-X.

2. Cappuccio FP, Miller MA. Cardiovascular disease and hypertension in subSaharan Africa: burden, risk and interventions. Intern Emerg Med. 2016;11(3): 299-305. https://doi.org/10.1007/s11739-016-1423-9.

3. Manning, E.B., et al., Dietary patterns in urbanised blacks. A study in Guguletu, Cape Town, 1971. S Afr Med J, 1974. 48(12): p. 485-498.

4. Keates AK, Mocumbi AO, Ntsekhe M, Sliwa K, Stewart S. Cardiovascular disease in Africa: epidemiological profile and challenges. Nat Rev Cardiol. 2017;14(5):273-93. https://doi.org/10.1038/nrcardio.2017.19.

5. Gomez-Olive FX, et al. Regional and sex differences in the prevalence and awareness of hypertension: an H3Africa AWI-Gen study across 6 sites in subSaharan Africa. Glob Heart. 2017;12(2):81-90. https://doi.org/10.1016/j.ghea rt.2017.01.007

6. Collaborators, G.C.o.C.o.D., Global, regional, and national age-sex specific mortality for 264 causes of death, 1980-2016: a systematic analysis for the Global Burden of Disease Study 2016. Lancet, 2017. 390(10100): p. 11511210.

7. Haas $A D$, et al. Retention and mortality on antiretroviral therapy in subSaharan Africa: collaborative analyses of HIV treatment programmes. J Int AIDS Soc. 2018;21(2):e25084.

8. Kiplagat J, Mwangi A, Keter A, Braitstein P, Sang E, Negin J, et al. Retention in care among older adults living with HIV in western Kenya: A retrospective observational cohort study. PLoS One. 2018;13(3):e0194047. https://doi.org/10.1371/journal.pone.0194047.

9. Labhardt ND, Balo JR, Ndam M, Manga E, Stoll B. Improved retention rates with low-cost interventions in hypertension and diabetes management in a rural African environment of nurse-led care: a cluster-randomised trial. Trop
Med Int Health. 2011;16(10):1276-84. https://doi.org/10.1111/j.1365-3156.2 011.02827.x.

10. Labhardt ND, Balo JR, Ndam M, Grimm JJ, Manga E. Task shifting to nonphysician clinicians for integrated management of hypertension and diabetes in rural Cameroon: a programme assessment at two years. BMC Health Serv Res. 2010;10(1):339. https://doi.org/10.1186/1472-6963-10-339.

11. Kengne AP, Fezeu L, Sobngwi E, Awah PK, Aspray TJ, Unwin NC, et al. Type 2 diabetes management in nurse-led primary healthcare settings in urban and rural Cameroon. Prim Care Diabetes. 2009;3(3):181-8. https://doi.org/1 0.1016/j.pcd.2009.08.005

12. Birungi J, et al. Integrating health services for HIV-infection, diabetes and hypertension in sub Saharan Africa: a cohort study. Liverpool: Liverpool School of Tropical Medicine; 2020.

13. United Nations. World Population Prospects. 2019 [cited 20201 March 2020]; Rev. 1:[Online Edition]. Available from: https://population.un.org/wpp/ Download/Standard/Interpolated/.

14. Van Hout MC, et al. Strengthening integration of chronic care in Africa: protocol for the qualitative process evaluation of integrated HIV, diabetes and hypertension care in a cluster randomised controlled trial in Tanzania and Uganda. BMJ Open. 2020;10(10):e039237. https://doi.org/10.1136/ bmjopen-2020-039237.

15. Dudley, L. and P. Garner, Strategies for integrating primary health services in low- and middle-income countries at the point of delivery. Cochrane Database Syst Rev, 2011. 2011(7): Cd003318.

16. Niessen LW, Mohan D, Akuoku JK, Mirelman AJ, Ahmed S, Koehlmoos TP, et al. Tackling socioeconomic inequalities and non-communicable diseases in low-income and middle-income countries under the Sustainable Development agenda. Lancet. 2018;391(10134):2036-46. https://doi.org/10.1 016/S0140-6736(18)30482-3.

17. Abbas ZG. Diabetic foot - an African perspective. JSM Foot and Ankle. 2016; $1(1): 1005$

18. Cavanagh P, Attinger C, Abbas Z, Bal A, Rojas N, Xu ZR. Cost of treating diabetic foot ulcers in five different countries. Diabetes Metab Res Rev. 2012;28(Suppl 1):107-11. https://doi.org/10.1002/dmrr.2245.

19. Danmusa UM, Terhile I, Nasir IA, Ahmad AA, Muhammad HY. Prevalence and healthcare costs associated with the management of diabetic foot ulcer in patients attending Ahmadu Bello University Teaching Hospital, Nigeria. Int J Health Sci (Qassim). 2016;10(2):219-28.

20. Mutyambizi C, Pavlova M, Chola L, Hongoro C, Groot W. Cost of diabetes mellitus in Africa: a systematic review of existing literature. Global Health. 2018;14(1):3. https://doi.org/10.1186/s12992-017-0318-5.

21. Ponatshego, P.L., Lawrence D.S., Youssouf N., Molloy S.F., Alufandika M., Bango F., Boulware D.R., Chawinga C., Dziwani E., Gondwe E., Hlupeni A., Hosseinipour M.C., Kanyama C., Meya D.B., Mosepele M., Muthoga C., Muzoora C.K., Mwandumba H., Ndhlovu C.E., Rajasingham R., Sayed S., Shamu S., Tsholo K., Tugume L., Williams D., Maheswaran H., Shiri T., Boyer-Chammard T., Loyse A., Chen T., Wang D., Lortholary O., Lalloo D.G., Meintjes G., Jaffar S., Harrison T.S., Jarvis J.N., Niessen L.W., AMBlsome Therapy Induction OptimisatioN (AMBITION): high dose AmBisome for cryptococcal meningitis induction therapy in sub-Saharan Africa: economic evaluation protocol for a randomised controlled trial-based equivalence study. BMJ Open, 2019. 9(4): p. e026288, DOl: https://doi.org/10.1136/bmjopen-2018-026288.

22. Drummond M, Sculpher M. Common methodological flaws in economic evaluations. Med Care. 2005;43(7 Suppl):5-14. https://doi.org/10.1097/01.mlr. 0000170001.10393.b7.

23. Husereau D, Drummond M, Petrou S, Carswell C, Moher D, Greenberg D, et al. Consolidated Health Economic Evaluation Reporting Standards (CHEERS) statement. BMJ. 2013;346(mar25 1):f1049. https:/doi.org/10.1136/bmj.f1049.

24. Barber JA, Thompson SG. Analysis of cost data in randomized trials: an application of the non-parametric bootstrap. Stat Med. 2000;19(23):3219-36. https://doi.org/10.1002/1097-0258(20001215)19:23<3219::AID-SIM623>3.0.CO;2-P.

25. Banker RD, Charnes A, Cooper WW. Some models for estimating technical and scale inefficiencies in data envelopment analysis. Management Science. 1984;30(9):1078-92. https://doi.org/10.1287/mnsc.30.9.1078.

26. Alatawi A, Ahmed S, Niessen L, Khan J. Systematic review and meta-analysis of public hospital efficiency studies in Gulf region and selected countries in similar settings. Cost Eff Resour Alloc. 2019;17(1):17. https://doi.org/10.1186/ s12962-019-0185-4.

27. Alatawi AD, Niessen LW, Khan JAM. Efficiency evaluation of public hospitals in Saudi Arabia: an application of data envelopment analysis. BMJ Open. 2020;10(1):e031924. https://doi.org/10.1136/bmjopen-2019-031924. 
28. Chang H, Hawley NL, Kalyesubula R, Siddharthan T, Checkley W, Knauf F, et al. Challenges to hypertension and diabetes management in rural Uganda: a qualitative study with patients, village health team members, and health care professionals. Int J Equity Health. 2019;18(1):38. https://doi.org/1 0.1186/s12939-019-0934-1.

29. Shade SB, Osmand T, Luo A, Aine R, Assurah E, Mwebaza B, et al. Costs of streamlined HIV care delivery in rural Ugandan and Kenyan clinics in the SEARCH Studys. AIDS. 2018;32(15):2179-88. https://doi.org/10.1097/QAD. 0000000000001958.

30. Barnett K, Mercer SW, Norbury M, Watt G, Wyke S, Guthrie B. Epidemiology of multimorbidity and implications for health care, research, and medical education: a cross-sectional study. Lancet. 2012;380(9836):37-43. https://doi. org/10.1016/S0140-6736(12)60240-2

31. Prados-Torres A, Calderón-Larrañaga A, Hancco-Saavedra J, Poblador-Plou B, van den Akker M. Multimorbidity patterns: a systematic review. J Clin Epidemiol. 2014;67(3):254-66. https://doi.org/10.1016/j.jclinepi.2013.09.021.

32. Maher D, Ford N, Unwin N. Priorities for developing countries in the global response to non-communicable diseases. Global Health. 2012;8(1):14. https://doi.org/10.1186/1744-8603-8-14.

33. Samb B, Desai N, Nishtar S, Mendis S, Bekedam H, Wright A, et al. Prevention and management of chronic disease: a litmus test for healthsystems strengthening in low-income and middle-income countries. Lancet. 2010;376(9754):1785-97. https://doi.org/10.1016/S0140-6736(10)61353-0.

34. von Elm E, Altman DG, Egger M, Pocock SJ, Gøtzsche PC, Vandenbroucke $J \mathrm{P}$, et al. The Strengthening the Reporting of Observational Studies in Epidemiology (STROBE) statement: guidelines for reporting observational studies. J Clin Epidemiol. 2008;61(4):344-9. https://doi.org/10.1016/j.jclinepi.2 007.11.008.

35. Shayo $E$, et al. Ethical issues in intervention studies on the prevention and management of diabetes and hypertension in sub-Saharan Africa. BMJ Glob Health. 2020;5(7):e002193.

36. RESPOND consortium, INTE-AFRICA programme. 2019 04/01/2021 [cited 2021 25/05/2021]; HORIZON 2020. Available from: https://inteafrica.org/a bout/.

\section{Publisher's Note}

Springer Nature remains neutral with regard to jurisdictional claims in published maps and institutional affiliations.

Ready to submit your research? Choose BMC and benefit from:

- fast, convenient online submission

- thorough peer review by experienced researchers in your field

- rapid publication on acceptance

- support for research data, including large and complex data types

- gold Open Access which fosters wider collaboration and increased citations

- maximum visibility for your research: over $100 \mathrm{M}$ website views per year

At $\mathrm{BMC}$, research is always in progress.

Learn more biomedcentral.com/submissions 